\title{
RECURRENT FEATURE GROUPING AND Classification MODEL FOR ACTION MODEL PREDICTION IN CBMR
}

\author{
Vinoda Reddy ${ }^{1}$, P.Suresh Varma ${ }^{2}$, A.Govardhan ${ }^{3}$ \\ ${ }^{1}$ Associate Professor \& HOD, CSE Dept., SIT, Gulbarga, Karnataka, India. \\ ${ }^{2}$ Principal, University College of Engineering,AdikaviNannaya University, Rajahmundry, \\ A.P, India \\ ${ }^{3}$ Principal, University College of Engineering, JNTUH, Hyderabad, Telangana, India.
}

\begin{abstract}
:
Content based retrieval has an advantage of higher prediction accuracy as compared to tagging based approach. However, the complexity in its representation and classification approach, results in lower processing accuracy and computation overhead. The correlative nature of the feature data are un-explored in the conventional modeling, where all the data features are taken as a set of feature values to give a decision. The recurrent feature class attribute is observed for the feature regrouping in action model prediction. In this paper a co-relative information, bounding grouping approach is suggested for action model prediction in CBMR application. The co-relative recurrent feature mapping results in faster retrieval process as compared to the conventional retrieval system.
\end{abstract}

\section{KEY WORDS:}

Recurrent feature mapping, classification, action model prediction, CBMR.

\section{INTRODUCTION}

With the improvements in new mode of data exchange approaches, sharing of data from one location to other has become easier. So, as the updation to a remote database is also improved. The more, the collection of data, more accurate the retrieval accuracy is. However, the process of retrieval depends on the passed query and the defining tag information in the database. In this direction, content based Multimedia retrieval (CBMR) has emerged as a retrieval system for multimedia data.Nowadays as large multimedia data sets such as audio, speech, text, web, image, video and combinations of several types are becoming increasingly available and are almost unstructured or semi-structured data by nature, which makes it difficult for human beings to extract the information without powerful tools. This drives the need to develop data mining techniques that can work on all kinds of data such as documents, images, and signals. [1] explores on survey of the current state of multimedia data mining and knowledge discovery, data mining efforts aimed at multimedia data, current approaches and well known techniques for mining multimedia data. [2] Provides a survey of the state-of-the-art in the field of Visual Information Retrieval (VIR) systems, particularly Content-Based Visual Information Retrieval (CBVIR) systems. It presents the main concepts and system design issues, reviews many research prototypes and commercial solutions currently available and points out promising research directions in this area. Content-based information retrieval of multimedia data is a great and attractive challenge which raises numerous research activities. As multimedia data become ubiquitous in our daily lives, information retrieval systems have to adapt their retrieval DOI: $10.5121 /$ ijdkp.2017.7605 
performance to different situations in order to efficiently satisfy the users' information needs anytime and anywhere.

To enhance the content-based multimedia information retrieval process, [3] proposes an efficient similarity measure based on flexible feature representations. It also defines the similarity model for content-based information retrieval and show its feasibility on real world multimedia data. Recently, information retrieval for text and multimedia content has become an important research area. Content based retrieval in multimedia is a challenging problem since multimedia data needs detailed interpretation from pixel values. [4] Proposed an overview of the content based retrieval is presented along with the different strategies in terms of syntactic and semantic indexing for retrieval. The matching techniques used and learning methods employed are also analyzed, and key directions for future research are also presented. Multimedia database management systems can be seen as storage and retrieval systems, in which large volumes of media objects are created, indexed, modified, searched and retrieved. There are two types of retrievals: navigation through database to locate the desired data and database querying that finds the desired data associatively, attribute-based, or content-based. Query processing in multimedia databases has the following stages: query specification and refining, query processing (compiling, optimizing, and executing), generation of relevancy ranked results, and relevance feedback. In content-based retrieval systems, the collections of multimedia objects are stored as digitized representations. The queries are characterized by fuzziness and ambiguity. Therefore, the user will not get from the beginning what s/he wants. Metrics (precision, recall) and indexation and content-based retrieval techniques can help, but the user is the one who will have the last word in appreciation of a query results. Many multimedia applications, such as the World-Wide-Web (WWW), video-on-demand (VOD), and digital library, require strong support of a video database system. In [6], a survey was done on various approaches to content-based video data retrieval. Compared with traditional keywordbased data retrieval, content-based retrieval provides a flexible and powerful way to retrieve video data. It also discusses three approaches in depth. First, since video segmentation is an elementary task in video index construction, we discuss an approach to detect shot changes for video segmentation, which is based on the processing of MPEG compressed video data. In [7] the current state-of-the-art in Relevance Feedback was discussed as seen from content-based image retrieval point of view and recommend a novel approach for the future. The large and growing amount of digital data, and the development of the Internet highlight the need to develop sophisticated access methods that provide more than just simple text-based queries. Many programs have been developed with complex mathematics algorithms to allow the transformation of image or audio data in a way that enhances searching accuracy. However it becomes difficult when dealing with large sets of multimedia data. [8] Proposes and demonstrates a Content Based Multimedia Retrieval System (CBMRS). The proposed CBMRS includes both video and audio retrieval systems. The Content Based Video Retrieval System (CBVRS) based on DCT and clustering algorithms. The audio retrieval system based on Mel-Frequency Cepstral Coefficients (MFCCs), the Dynamic Time Warping (DTW) algorithm and the Nearest Neighbor (NN) rule. With the development of multimedia data types and available bandwidth there is huge demand of video retrieval systems, as users shift from text based retrieval systems to content based retrieval systems.

Selection of extracted features play an important role in content based video retrieval regardless of video attributes being under consideration. These features are intended for selecting, indexing and ranking according to their potential interest to the user. Good features selection also allows the time and space costs of the retrieval process to be reduced. [9] Reviews the interesting features that can be extracted from video data for indexing and retrieval along with similarity 
measurement methods. It also identifies present research issues in area of content based video retrieval systems. Image retrieval has been popular for several years. There are different system designs for content based image retrieval (CBIR) system. [10] Propose a novel system architecture for CBIR system which combines techniques include content-based image and color analysis, as well as data mining techniques. To our best knowledge, this is the first time to propose segmentation and grid module, feature extraction module, K-means and k-nearest neighbor clustering algorithms and bring in the neighborhood module to build the CBIR system. Concept of neighborhood color analysis module which also recognizes the side of every grids of image is first contributed in [10] only. The spatial, temporal, storage, retrieval, integration, and presentation requirements of multimedia data differ significantly from those for traditional data. A multimedia database management system provides for the efficient storage and manipulation of multimedia data in all its varied forms. [11] looks into the basic nature of multimedia data, highlight the need for multimedia DBMSs, and discuss the requirements and issues necessary for developing such systems. [12] Presents a query processing strategy for the content-based video query language named CVQL. By CVQL, users can flexibly specify query predicates by the spatial and temporal relationships of the content objects. The query processing strategy evaluates the predicates and returns qualified videos or frames as results. A promising approach is based on using the audio layer of a lecture recording to obtain information about the lecture's contents [13] present an indexing method for computer science courses based on their existing recorded videos. The transcriptions from a speech-recognition engine (SRE) are sufficient to create a chain index for detailed browsing inside a lecture video. The index structure and the evaluation of the supplied keywords are presented. Content sharing networks, such as YouTube, contain traces of both explicit online interactions (such as likes, comments, or subscriptions), as well as latent interactions (such as quoting, or remixing, parts of a video).[14] propose visual memes, or frequently re-posted short video segments, for detecting and monitoring such latent video interactions at scale. Visual memes are extracted by scalable detection algorithms that we develop, with high accuracy. It further augments visual memes with text, via a statistical model of latent topics. [14] models content interactions on YouTube with visual memes, defining several measures of influence and building predictive models for meme popularity. Main goal of [15] is to show the improvement of using a textual pre-filtering combined with an image re-ranking in a Multimedia Information Retrieval task. The define three step based retrieval processes and a wellselected combination of visual and textual techniques help the developed Multimedia Information Retrieval System to overcome the semantic gap in a given query.

In [15] five different late semantic fusion approaches are discussed and experimented in a realistic scenario for multimedia retrieval like the one provided by the publicly available Image CLEF Wikipedia Collection. As digital video databases grow, so grows the problem of effectively navigating through them. [16] Propose a novel content-based video retrieval approach to searching such video databases, specifically those involving human actions, incorporating spatio-temporal localization. It outline a novel, highly efficient localization model that first performs temporal localization based on histograms of evenly spaced time-slices, then spatial localization based on histograms of a 2-D spatial grid. It further argue that our retrieval model, based on the aforementioned localization, followed by relevance ranking, results in a highly discriminative system, while remaining an order of magnitude faster than the current state of- theart method. [17] propose scalable solutions to learning query-specific distance functions by 1) adopting a simple large-margin learning framework, 2) using the query-logs of text-based image search engine to train distance functions used in content-based systems. Recent research shows that multimedia resources in the wild are growing at a staggering rate. The rapid increase number 
of multimedia resources has brought an urgent need to develop intelligent methods to organize and process them. In [18], the semantic link network model is used for organizing multimedia resources. A whole model for generating the association relation between multimedia resources using semantic link network model is proposed. The definitions, modules, and mechanisms of the semantic link network are used in the proposed method. The integration between the semantic link network and multimedia resources provides a new prospect for organizing them with their semantics. The semantic gap between low-level visual features and high-level semantics is a wellknown challenge in content-based multimedia information retrieval. With the rapid popularization of social media, which allows users to assign tags to describe images and videos, attention is naturally drawn to take advantage of these metadata in order to bridge the semantic gap. [19] proposes a Sparse Linear Integration (SLI) model that focuses on integrating visual content and its associated metadata, which are referred to as the content and the context modalities respectively, for semantic concept retrieval. An optimization problem is formulated to approximate an instance using a sparse linear combination of other instances. The difference between them is considered as the error to minimize. In [20] first present a global image data model that supports both metadata and low-level descriptions of images and their salient objects. This allows making multi-criteria image retrieval. Then, it presents an image data repository model that captures all the data described in the model and permit to integrate heterogeneous operations in a DBMS. In particular, content-based operations (content-based join and selection) in combination with traditional ones can be carried out using our model. However, the conventional approaches lack the correlative property of feature matching resulting in lower processing accuracy and increase in overhead. To eliminate these features, in this paper an new feature recurrent mapping model is proposed. To present the suggested approach, the rest of the paper is presented in 7 sections. The CBMR model and its feature representation is outlined in section 2. Section 3 outlines the feature regrouping approach. The suggested approach of recurrent feature regrouping is presented in section 4 . Section 5 illustrates the result obtained.

\section{CBMr Model And Feature RePresentation [22]}

For the extraction of features in the action model prediction, the developed past work [22] of multi kernel dimensional reduction technique is used. The proposed multimedia retrieval system consists of two phases. In the training phase, the training process is developed for database which facilitates the updation of various multimedia features extracted from different video samples. In the test phase, the test video sample is processed for feature extraction and the SVM classifier using the training features are extracted from the database are compared with the features, matching the result of the test video multimedia feature set taken as multimedia features.

The Multimedia retrieval system proposed is divided into four operational phases:

I) Pre-processing

2) feature extraction using 2D - Gabor filter and histogram

3) Feature vector dimensionality reduction MLK-DR.

4) multimedia retrieval system using SVM classifier.

The proposed approach is a multimedia video retrieval system that is used to estimate multimedia information's from the dataset. Multimedia video from database sources are acquired and the retrieval system process the video input which is then further processed as multimedia video feature set. For a given multimedia video sample using the histogram feature to estimate the feature estimate approach and multi-linear dimension reduction (ML-DR) is used in association 
with a 2D-Gabor filter. The Input multimedia video preprocessing phase, process on the input video data read from the database and process through the images. The samples are then resized, cropped and converted to gray scale for feature representation. After preprocessing, multimedia video goes through feature extraction process. At this stage, all possible orientations of Gabor filter was applied to remove all possible variations. Here, the entire Gabor filter is applied on eight orientations and histogram feature set is derived. In the next step, the extracted features are used to reduce the dimensionality using multi-linear approach to reduce feature vectors. A SVM classifier using these featurevectors are used with the database to compare and match the classes for classifying the multimedia information.

The proposed algorithm for the system is as follows.

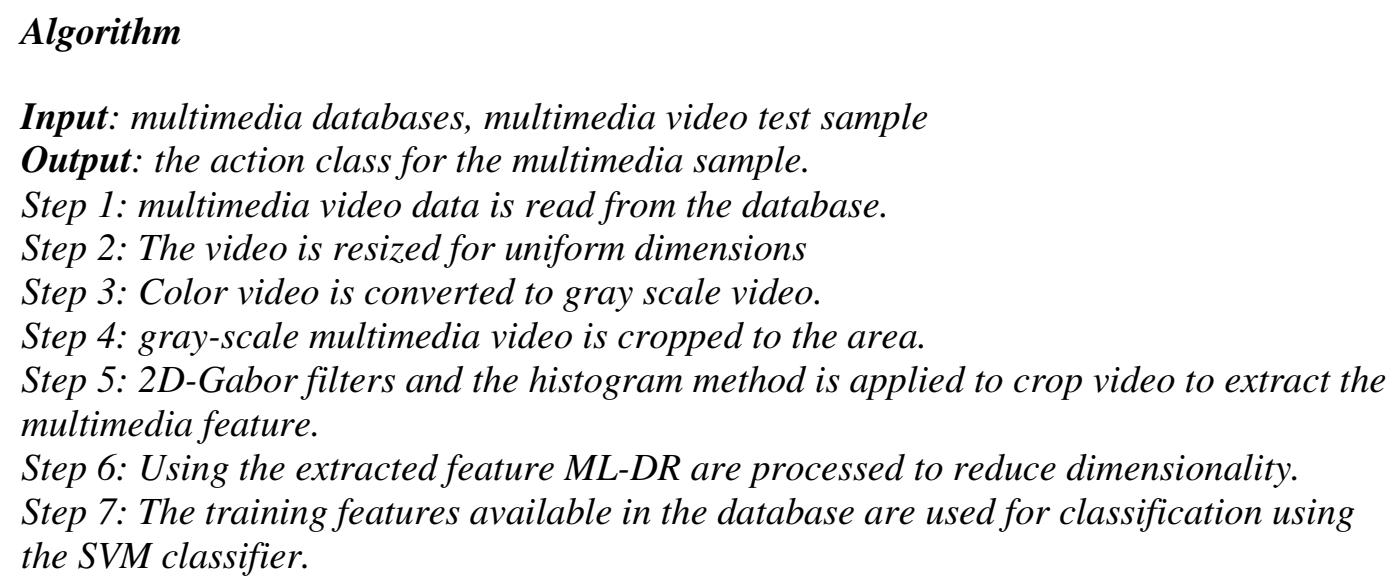

In the Multi-linear dimensionality reduction ML-DR approach the features are transformed to the multi-linear subspace that extracts the multi-dimensional features from the database. ML-DR, which operated in linear dimension for expansion. While ML-DR operated directly on the twomode processing the data are processed through multi-functional objectives. Video filtering using Gabor filter and feature extraction for dimensionality reduction in ML-DR results in output for dimensionally reduced feature multimedia dataset which are derived from projection matrix.

\section{Feature Regrouping ApProach}

Co-clustering can be simply considered as a simultaneous clustering of more properties of the dataset. Co-clustering produces a set of clusters of original data and also a set of clusters of their property. Unlike other clustering algorithms, co-clustering also defines a clustering criterion and then optimizes it. In a nutshell, co-clustering finds out the subsets of attributes and their properties simultaneously of a data matrix using a specified criterion. From the summarization point of view, co-clustering provides significant benefits. Basically, there are two types of coclustering approaches, Block co-clustering and Information Theoretic co-clustering. Among the defined co-clustering logic, the Information Theoretic Co-clustering (ITCC) approach was proved to be optimal [21]. In the information theoretic co-clustering was based on Bergman divergence. This ITCC tries to reduce the loss of information in the approximation of a data matrix X. The reduction is data loss can be minimized through a predefined distance called Bergman divergence function. For a given co-clustering $(\mathrm{R}, \mathrm{C})$ and a matrix approximation scheme $\mathrm{P}$, a class of random variables which store the properties of attribute data matrix $\mathrm{X}$ is defined. The objective function 
tries to reduce the attributes loss on the approximation of $\hat{X}$ for a co-clustering $\mathrm{R}, \mathrm{C}$. The Bregman information of $\mathrm{X}$ is defined by [21]:

$$
B I_{\phi}(X)=E\left[\log \frac{X}{E[X]}\right]
$$

Here, the matrix approximation scheme is defined by the expected value and the Bergman divergence $d_{\phi}$ for an optimal co-clustering as follows

$$
(R, C)=\arg \min E\left[d_{\phi}(X, \hat{X})\right]
$$

Here, the Bergman divergence was directly related with Euclidean distance and can be expressed as

$$
d_{\phi}=\left(x_{1}-x_{2}\right)^{2}
$$

Here, the Bergman divergence was evaluated by just calculating the Euclidean distance between the attributes magnitude values. The Euclidean distance will give the difference between the magnitudes of attribute values. Though this approach provides better diagnosis accuracy, it is almost equal to the diagnosis accuracy of sub-clustering approach. This approach is not considering the divergence of attribute properties. If the co-clustering was done by considering the divergence of attribute property, the diagnosis accuracy will be increased further. The complete detail about the proposed approach is given in next section.

\section{RECURRENT FEATURE Grouping (RFG) AND Classification}

In the approach of co-clustering the data from the selected sub-clusters of dataset, the approach of conventional co-clustering using ITCC was observed to be effective. However, in the approach of co-clustering operation following ITCC, the clustering are made based on the divergence factor of two observations $\left(x_{1}, x_{2}\right)$. the divergence factor is computed using the Euclidian distance based approach, where two of the magnitudal components are compared to obtain a distance factor. The convergence is observed when the two observations satisfy a minimum divergence criterion. Wherein the approach results in a optimal co-clustering of data, the relational property of the data elements are not explored. However the approach of ITCC does not consider nature of attribute variation for clustering. Though the data type attribute results in proper defining of nature of variation for the observing attribute, a Euclidian approach is not appropriate in clustering. Hence in this proposed method, a weighted ITCC coding for co-clustering of data type is presented. The approach of co-clustering using weight factor is defined by allocating a weight value to the distinct class attributes in the sub cluster. Let $w_{i}$ be the allocated weight for each sub cluster $C_{i}$, where the sub cluster are derived using the method of MLKDR selected feature set, clustered using the data type property with 5 distinct class of effect,

$$
C_{i} \in(R, W, J)
$$

Where the class attributes is defined as, 
Table 1: Class label attribute to distinct classes formed

\begin{tabular}{|c|c|}
\hline Class Type $\left(\boldsymbol{C}_{\boldsymbol{i}}\right)$ & Associated weight $\left(\boldsymbol{w}_{\boldsymbol{i}}\right)$ \\
\hline Running & 1 \\
\hline Walking & 2 \\
\hline Jumping & 3 \\
\hline Bending & 4 \\
\hline
\end{tabular}

The allocated weights were assigned based on the action effect of each class attribute. The class with running condition is given weight ' 1 '. In the process of mining the dataset for a information retrieval, the given query details are mined over the sub cluster dataset based on the correlation feature set in the recurrent mapped feature sets, derived as outlined in previous section. To optimize the mining performance, co-clustering approaches were introduced. The dataset is clustered into sub clusters based on the criterion of Bergman divergence Information $I_{\varnothing}(X)$, satisfying the convergence problem,

$$
\arg \min \left(E\left[d_{\emptyset}(X, \tilde{X}]\right)\right.
$$

Where $X \in C_{i}$ and $\tilde{X}$ is the new co-cluster formed for the sub cluster $C_{i}$. Here, $d_{\emptyset}$ is defined as the divergence operator given by the Euclidian distance of the two observing element $\left(x_{1}, x_{2}\right)$,

$$
d_{\emptyset}\left(x_{1}, x_{2}\right)=\left(x_{1}, x_{2}\right)^{2}
$$

Clustering based on the consideration of coefficient magnitudes results in co-clusters based on the observing values, the relational property among the values and the severity of the class values are however not been considered. As observed, continuous data types are more effective in decision deriving than the discrete data type, so an inclusion of type factor and integrating the co-clusters based on the class attribute leads to finer co-clustering of the dataset. In consideration to the stated approach, the weighted co-clustering computes an aggregative weight value of each of the class label $\left(w_{i}\right)$ given as,

$$
W=\sum_{i=1}^{n} w_{i}
$$

Where, $w_{i}$ is the weight value assigned to a class attribute for $n$ sub classes under observation. While considering a co-clustering of each class, the aggregated weight value is computed, and the co-clustering limit of $\frac{W_{C}}{N}$ is set. Where, $W_{c}$ is the total weight values assigned to each class attribute.

$$
W_{c}=\sum_{i=1}^{N} w_{i}
$$

where $\mathrm{N}$ is the number of classes.

The convergence problem is then defined by,

$$
\arg \min \left(E\left[d_{\varnothing}(X, \tilde{X})\right] \Rightarrow\left(\min (W)>\frac{W_{C}}{N}\right)\right.
$$

The solution to the convergence problem, is here defined by two objectives, with minimum estimated divergence based on Euclidian distance, subjected to the condition having a minimum aggregated weight value lower than half of the total class weight value. A limit of $\frac{W_{C}}{N}$ is 
considered, to have a co-clustering objective of minimum similar elements entering into a class, without breaking the relational property among the observing attribute. The process of the proposed weighted ITCC (W-ITCC) approach is illustrated as follow,

Let, $C_{1}, C_{2}, C_{3}, C_{4}$ be a sub cluster derived from the selection approach of action model selection process with data type attribute. The associated weight for each of the class be $W_{1}, W_{2}, W_{3}, W_{4}$ assigned with the value of $\{1,2,3,4\}$. For this set of dataset, to apply weighted co-clustering process the following step of operations are applied,

Step 1: The Bergman divergence for the two observing element is computed using (6).

Step 2 : For each divergence, $\mathrm{d}_{\varnothing}\left(\mathrm{x}_{1}, \mathrm{x}_{2}\right)$, the convergence criterion defined by (5) is computed.

Step 3 : The aggregative sum weighted value for the observing class is then computed using (7).

Step 4: The convergence criterion is then checked for the obtained aggregated weight value following (9).

Step 5 : The above steps are repeated for all sub classes to co-cluster.

In the considered case, the total weight

While co-clustering the elements of the entire sub class is obtained by,

$$
W_{c}=\sum_{i=1}^{N} w_{i}=10 .
$$

Now, for the clustering of two sub classes, $C_{1}, C_{2}$ and $C_{1}, C_{4}$, the aggregative weight sum is obtained as,

$$
W_{12}=3 \text { and } W_{14}=7 \text {. }
$$

Considering convergence problem of (9), the limiting value is $10 / 4=2.5$.

In this case $W_{14}>\frac{W_{c}}{N}$ hence not clustered in the same group, even though the divergence criterion is satisfied. This results in co-clustering of sub-classes with highest similarity into one class. As, could be observed, class- 1 and class- 4 are of more dissimilarity than class 1 and 2 . The allocated weight values governed the class relation and the limiting boundness of the total weight value prevents the wrong clustering of the sub-class. The operational algorithm for the suggested weighted co-clustering approach is as illustrated below,

\section{EXPERIMENTAL RESULTS}

The proposed system is developed with Matlab tools and Weizmann data set [7] is tested over. PCA-based feature dimensions reduction is compared with a comparative analysis of the proposed MLK-DR. the simulation is carried out to evaluate the performance of the proposed approach. Weizmann dataset simulation for features extraction for which Histogram is computed as MI-HIST [21] is set forth in the calculation. Figure.1 illustrates the test dataset is shown as derived from [22] 
International Journal of Data Mining \& Knowledge Management Process (IJDKP) Vol.7, No.5/6, November 2017
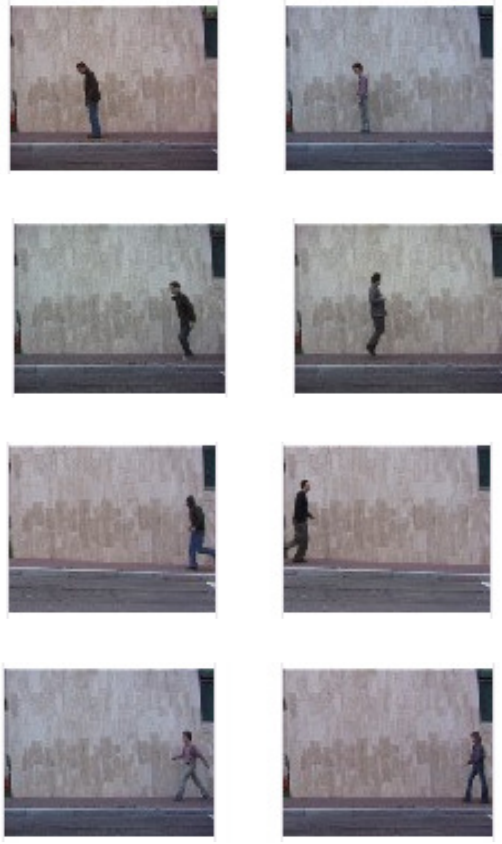

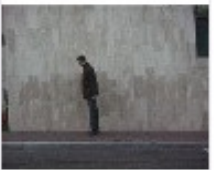

(a)

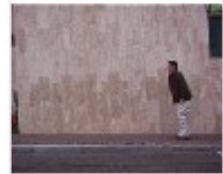

(b)

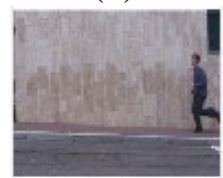

(c)

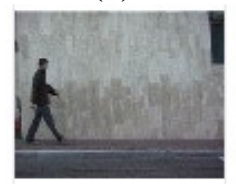

(d)
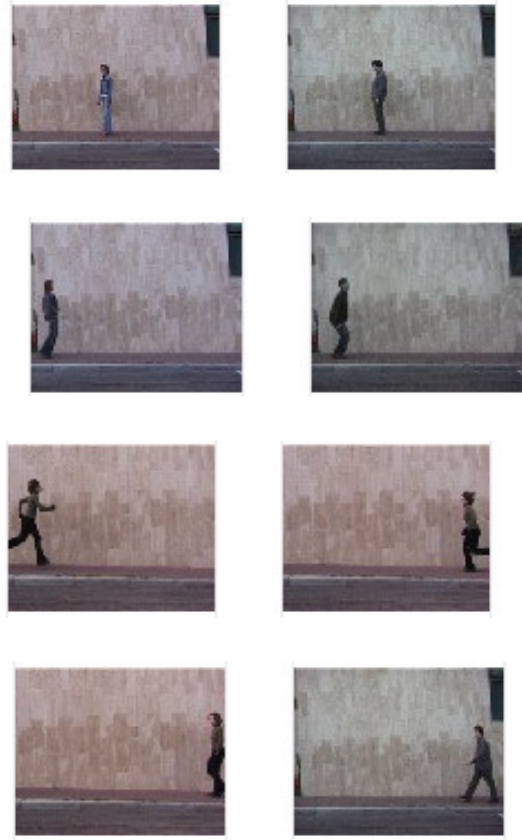

Fig 1: Dataset with (a) Bending (B), (b) Jumping (J), (c) Running (R) and (d) Walking (W) sample

The samples are captured a 180x144 resolution, using static camera with a homogenous outdoor background.The processing test sample having a running action is illustrated in Fig 2.

Orignal Sample

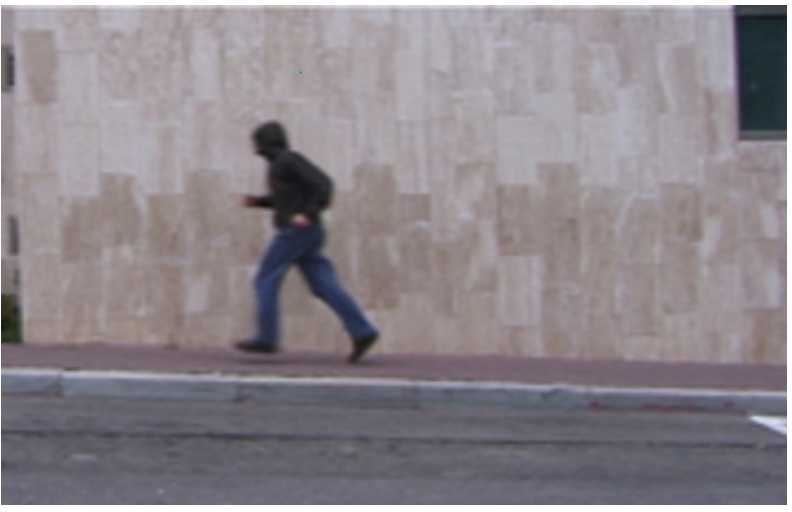

Fig 2: Test sample having Running action

The obtained features using MI-HIST [21] is presented in table 2.

Table 2. Comparative Analysis of HoG [22], HIST [23] and proposed MI-HIST [21] for running sample

\begin{tabular}{|l|c|c|c|}
\hline \multicolumn{1}{|c|}{ Observations of Techniques } & HoG [22] & HIST [23] & MI-HIST[21] \\
\hline Original Sample Size & 478720 & 478720 & 478720 \\
\hline Redundant coefficients & 436582 & 457581 & 464260 \\
\hline $\begin{array}{l}\text { HIST Features for Motion } \\
\text { components }\end{array}$ & 42138 & 21139 & $\mathbf{1 4 4 6 0}$ \\
\hline
\end{tabular}


MI-HIST Histogram features are used for dimensionality reduction, where PCA, LDA and MLKDR are applied over it. In the PCA-based dimensionality reduction technique, the extracted features and the mean normalized $\mathrm{K}$ principal features are selected. On the count of selected facilities where facilities are used for the inter-class correlation, where LDA coding is applied for least minimum feature count. However, PCA and LDA dimensionality reduction process for twodimensional coding. To further reduce the dimensions of the features, MLK-DR was implemented. To validate the observation, this section illustrates the details about the result analysis. The performance of proposed approach was verified through accuracy. The accuracy of the proposed approach was measured using the standard confusion metrics. The metrics are listed as True Positive (TP), False Positive (FP), True Negative (TN), False negative (FN). The complete analysis was carried out on all the classes of action model. Initially, the TP denotes the true people correctly identified as true, FP denotes the falseclass incorrectly identified as true, The accuracy at each and every stage was evaluated by the following mathematical expression,

$$
\text { Accuracy }=\frac{T P+T N}{T P+T N+F P+F N}
$$

Table.3. Accuracy Comparison for proposed approach with earlier approaches under all classes compute the measured parameters, a mathematical expressions used are defined as, The sensitivity is measured as the ratio of true positive (TP) to the sum of true positive (TP) and false negative $(\mathrm{FN})$.

$$
\text { Sensitivity }=\frac{T P}{T P+F N}
$$

The specificity is measured as the ratio true negative (TN) to the sum of true negative (TN) and false positive (FP).

$$
\text { Specificity }=\frac{T N}{T N+F P}
$$

Precision is the ratio of TP to sum of TP and FP while recall is the ratio of TP to sum of TP and FN. The following expressions give precision and recall measurements

$$
\begin{aligned}
& \text { Recall }=\frac{T P}{T P+F N} \\
& \text { Precision }=\frac{T P}{T P+F P}
\end{aligned}
$$

F-measure is the combined measure of precision and recall. F-measure is also called as balanced F-score, the expression is as

$$
\text { F_measure }=\frac{2 * \text { Recall.Precision }}{\text { Recall }+ \text { Precision }}
$$

Table 3.Parametric evaluation of the developed system for processing efficiency

\begin{tabular}{|c|c|c|c|c|c|}
\hline \multirow{2}{*}{ Input } & \multicolumn{5}{|c|}{ Accuracy (\%) } \\
\cline { 2 - 6 } & PCA & LDA & MLK-DR & ITCC & RFG \\
\hline Running & 68.24 & 71.52 & 73.41 & 77.17 & 82.16 \\
\hline Walking & 67.25 & 70.42 & 72.15 & 78.52 & 84.68 \\
\hline Bending & 69.45 & 73.21 & 74.54 & 79.54 & 82.24 \\
\hline Jumping & 71.25 & 73.54 & 76.32 & 79.12 & $80.64 \%$ \\
\hline
\end{tabular}


Table.4. performance table for the developed approach

\begin{tabular}{|c|c|c|c|c|c|c|c|c|}
\hline & $\begin{array}{c}\text { Feature } \\
\text { Selection }\end{array}$ & Accuracy & Sensitivity & $\begin{array}{c}\text { Specificit } \\
\mathrm{y}\end{array}$ & $\begin{array}{c}\text { Reca } \\
\text { 1l }\end{array}$ & $\begin{array}{c}\text { Precisio } \\
\mathrm{n}\end{array}$ & $\begin{array}{c}\text { F- } \\
\text { Measure }\end{array}$ & Time \\
\hline \multirow{4}{*}{ Running } & MLK-DR & 41.3536 & 0.72 & 0.2853 & 0.72 & 0.5237 & 0.5873 & 0.3473 \\
\cline { 2 - 9 } & ITCC & 50.2252 & 0.58 & 0.2427 & 0.58 & 0.4217 & 0.4969 & 0.1380 \\
\cline { 2 - 9 } Walking & RFG & 70.0812 & 0.92 & 0.4588 & 0.91 & 0.6458 & 0.7558 & 0.1259 \\
\hline \multirow{4}{*}{ Bending } & MLK-DR & 51.5245 & 0.60 & 0.4052 & 0.60 & 0.5127 & 0.5450 & 0.2593 \\
\cline { 2 - 9 } & ITCC & 61.1340 & 0.88 & 0.5429 & 0.87 & 0.6546 & 0.7626 & 0.1479 \\
\cline { 2 - 9 } & RFG & 72.1554 & 0.72 & 0.2878 & 0.72 & 0.5237 & 0.5521 & 0.1625 \\
\hline \multirow{4}{*}{ jumping } & ITCC & 50.7636 & 0.72 & 0.2473 & 0.73 & 0.5256 & 0.5853 & 0.3587 \\
\cline { 2 - 9 } & RFG & 74.2540 & 0.90 & 0.5419 & 0.85 & 0.6636 & 0.7746 & 0.1473 \\
\cline { 2 - 9 } & MLK-DR & 52.7436 & 0.92 & 0.4758 & 0.88 & 0.6788 & 0.7698 & 0.1423 \\
\cline { 2 - 9 } & ITCC & 67.5640 & 0.90 & 0.5859 & 0.87 & 0.6426 & 0.7856 & 0.1780 \\
\hline
\end{tabular}

Computation Overhead, convergence time, cluster convergence, classification performance

\section{CONCLUSION}

A new approach of co-clustering logic based on the relational property of the data attributes and class values is presented. In the mining process for action model analysis is presented. The clustering operation based on data type property of continuous and discrete data is used. The subcluster formation results in optimal data clusters with recurrent and continuous feature selection, in consideration with its data type property. The data type property is observed to be a significant observation in appropriate clustering of dataset, and shown a higher performance in classification accuracy. The proposed co-clustering over this sub-cluster improves the performance accuracy in consideration to class type values assigned to each class level.

\section{REFERENCES}

[1] Manjunath T.N, Ravindra S Hegadi, Ravikumar.G.K, "A Survey on Multimedia Data Mining and Its Relevance Today", IJCSNS, 2010.

[2] Oge Marques and BorkoFurht, "Content-Based Visual Information Retrieval", Florida Atlantic University, USA, 2002.

[3] Christian Beecks Thomas Seidl, "Efficient Content-Based Information Retrieval: A New Similarity Measure for Multimedia Data", Symposium on Future Directions in Information Access, 2009.

[4] Ankush Mittal, "An Overview of Multimedia Content-Based Retrieval Strategies", Informatica 2006.

[5] CătălinaNegoiţă, Monica Vlădoiu, "Querying and Information Retrieval in Multimedia Databases", informatica 2006.

[6] Arbee L. P. Chen, Chih-Chin Liu, and Tony C. T. Kuo, "Content-based Video Data Retrieval", ROC, 1999. 
International Journal of Data Mining \& Knowledge Management Process (IJDKP) Vol.7, No.5/6, November 2017

[7] SunithaJeyasekhar and SihemMostefai, "Towards Effective Relevance Feedback Methods in ContentBased Image Retrieval Systems", International Journal of Innovation, Management and Technology, 2014.

[8] Yuk Ying Chung, Ka Yee Ng, Liwei Liu, "Design of a Content Based Multimedia Retrieval System", Int. Conf. on Circuits, Systems, Electronics, Control \& Signal Processing, 2006.

[9] B V Patel and B BMeshram, “Content Based Video Retrieval Systems” IJU, 2012.

[10] Ray-I Chang, Shu-Yu Lin, Jan-Ming Ho, Chi-Wen Fann , and Yu-Chun Wang, " A Novel Content Based Image Retrieval System using K-means/KNN with Feature Extraction”, ComSIS, 2012.

[11] Donald A. Adjeroh, "Multimedia Database Management Requirements and Issues", IEEE, 1997.

[12] Tony C. T. Kuo and Arbee L. P. Chen, "Content-Based Query Processing for Video Databases", IEEE 2000

[13] Stephan Repp, Andreas Groß, and ChristophMeinel, "Browsing within Lecture Videos Based on the Chain Index of Speech Transcription", IEEE, 2008.

[14] LexingXie, ApostolNatsev, "Tracking Large-Scale Video Remix in Real-World Events", IEEE 2013.

[15] XaroBenavent, Ana Garcia-Serrano, "Multimedia Information Retrieval Based on Late Semantic Fusion Approaches: Experiments on a Wikipedia Image Collection”, IEEE 2013.

[16] Ling Shao, "Efficient Search and Localization of Human Actions in Video Databases", IEEE 2014.

[17] Yushi Jing, Michele Covell, "Learning Query-Specific Distance Functions for Large-Scale Web Image Search", IEEE 2013.

[18] Chuanping Hu, "Semantic Link Network-Based Model for Organizing Multimedia Big Data", IEEE 2014.

[19] Qiusha Zhu, Mei-Ling Shyu, "Sparse Linear Integration of Content and Context Modalities for Semantic Concept Retrieval”, IEEE 2014.

[20] Solomon Atnafu, Richard Chbeir, Lionel Brunie, "Content-Based and Metadata Retrieval in Medical Image Database", IEEE 2002.

[21] Trupti S. Atre, K.V.Metre, "MIRS: Text Based and Content Based Image Retrieval”, IJESIT 2014.

[22] Vinoda Reddy, Dr.P.SureshVarma, Dr.A.Govardhan, "Multi-linear kernel mapping for feature dimension reduction in content based multimedia retrieval system", International Journal ofmultimedia and its application, Vol.8, No.2, 2016. 